\title{
Guideline Summary for Patients and Their Families
}

\author{
Werner J. Becker ${ }^{1}$ and Irene Worthington ${ }^{2}$ on behalf of the Canadian Headache \\ Society Acute Migraine Treatment Guideline Development Group
}

Can J Neurol Sci. 2013; 40: Suppl. 3 - S69-S72

This acute migraine treatment guideline is summarized here to provide information for patients with migraine and their families. Acute migraine medications are used to treat individual migraine attacks at the time of the attack. Most patients with migraine will use an acute medication, but patients with migraine, especially if they have frequent attacks, should also consider whether they could change lifestyle factors which might be making their headache more frequent (skipping meals, not enough sleep, etc), or whether they need a migraine preventive medication. Preventive (or prophylactic) medications are quite different from acute medications. Preventive medications are taken daily to reduce the frequency (number) of migraine attacks, while acute medications are used to reduce or stop the pain of a migraine attack once it has started. It is important that acute medications not be taken too often.

\section{OBJeCTIVE}

The objective of this guideline is to help in choosing the best acute medication for an individual with migraine, and to assist in using the chosen medication in the most effective way.

\section{Goals of acute migraine therapy}

The goal is for the patient to be pain free within two hours after treatment without medication side effects (or minimal side effects). Not all patients can achieve this goal with medications that are available at this time, but if patients are not reaching this goal, or not able to do their usual activities reasonably well at two hours, another medication should be tried if possible.

\section{Avoiding medication overuse}

Taking acute medications for migraine too often can result in more and more frequent headaches, even to the point where the patient is having headaches every day. This is called medication overuse headache.

To avoid medication overuse headache, ASA, antiinflammatory medications or NSAIDs (for example ibuprofen, naproxen, diclofenac) and acetaminophen should not be taken on more than 14 days per month. In the same way, triptans, ergots (such as ergotamine), opioids (for example codeine), or combination analgesics (for example acetaminophen with caffeine and codeine) should not be taken on more than nine days a month. Patients taking different acute medications on different days should limit their total use of acute medications to not more than nine days a month if one of their medications is a triptan, a combination analgesic, an ergotamine, or an opioid.

If patients have frequent migraine attacks, it is very useful to record use of acute medications, preferably with a headache diary. Preventive medications can also be helpful. If they reduce migraine attack frequency, patients will not need their acute medications as often.

Which acute migraine medications should be used?

Many medications have been proven to be helpful for the acute treatment of migraine attacks. These include:

1. The triptans: these are listed below with the usual doses used. The triptans can be taken with naproxen sodium $550 \mathrm{mg}$ if they are not effective enough by themselves. They can also be taken with a medication for nausea if necessary (metoclopramide $10 \mathrm{mg}$ or domperidone $10 \mathrm{mg}$ tablets).

a. Almotriptan $12.5 \mathrm{mg}$ tablets

b. Eletriptan $40 \mathrm{mg}$ tablets

c. Frovatriptan $2.5 \mathrm{mg}$ tablets

d. Naratriptan $2.5 \mathrm{mg}$ tablets

e. Rizatriptan $10 \mathrm{mg}$ tablets or wafers

f. Sumatriptan $100 \mathrm{mg}$ tablets, nasal spray $20 \mathrm{mg}$, and injection $6 \mathrm{mg}$

g. Zolmitriptan $2.5 \mathrm{mg}$ tablets or wafers, and nasal spray $5 \mathrm{mg}$

2. Several non-steroidal anti-inflammatory drugs (NSAIDs): these are listed below with the usual doses used. These medications can be taken with a medication for nausea if necessary (metoclopramide $10 \mathrm{mg}$, or domperidone $10 \mathrm{mg}$ tablets):

a. ASA $975-1,000 \mathrm{mg}$ in tablet form, or as an effervescent liquid (the effervescent liquid may work more quickly)

b. Ibuprofen $400 \mathrm{mg}$ in tablet form, or in a liquid-containing capsule which may work more quickly.

c. Naproxen sodium 500 - $550 \mathrm{mg}$ in tablet form

d. Diclofenac $50 \mathrm{mg}$ in tablet form or as a powder dissolved in water which works faster than the tablets.

3. Acetaminophen $1000 \mathrm{mg}$ in tablet form. Acetaminophen is helpful mainly for attacks of mild or moderate severity. It can be taken with a medication for nausea if necessary (metoclopramide $10 \mathrm{mg}$ or domperidone $10 \mathrm{mg}$ tablets).

\footnotetext{
From the ${ }^{1}$ University of Calgary and the Hotchkiss Brain Institute, Calgary, Alberta; ${ }^{2}$ Sunnybrook Health Sciences Centre, Toronto, Ontario, Canada. Received June 9, 2013. Final Revisions Submitted June 22, 2013. Correspondence to: W.J. Becker, Division of Neurology, 12th Floor, Foothills Hospital, 1403 29th St NW, Calgary, Alberta, T2N 2T9, Canada.
} 
4. Dihydroergotamine as a nasal spray (2 $\mathrm{mg})$ or a subcutaneous or intramuscular injection $(1 \mathrm{mg})$. This medication is not available as a tablet. It can be taken with a medication for nausea (metoclopramide $10 \mathrm{mg}$, or domperidone $10 \mathrm{mg}$ tablets).

5. Ergotamine (with caffeine) tablets: Although these can be helpful, they have many side effects, and the triptans usually work better.

Some medications that are sometimes used to treat migraine attacks are best avoided, if possible. They should not be used routinely. These include:

1. Painkillers (analgesics) that contain tramadol, usually in combination with acetaminophen.

2. Painkillers (analgesics) that contain codeine, usually in combination with acetaminophen or ASA and caffeine.

These medications are best avoided because they do not usually work very well for migraine, and they often lead to frequent use and medication overuse headache. If these medications are used, patients should monitor and record how often they use them with a headache diary.

These medications may be necessary is some patients, for example in those who have had a heart attack or stroke and cannot take triptans, or in patients who don't respond to NSAIDs or triptans. They may also be helpful for occasional use as a "rescue" medication when the patient's triptan fails to work.

There are several other medications that are sometimes used for migraine that are best avoided and used only in exceptional circumstances because of their tendency to lead to medication overuse headache and addiction. These include:

1. Butorphanol nasal spray and other strong opioids like oxycodone

2. Barbiturate (butalbital)-containing painkillers (analgesics), either with or without codeine.

In summary, the main medications for acute migraine treatment are the NSAIDs and the triptans. Acetaminophen can also be used for attacks of mild or moderate severity, and metoclopramide and domperidone can be added to help control nausea or vomiting.

\section{Treating early}

Patients with migraine attacks that are usually moderate or severe in intensity should be advised to take their acute medication early during their migraine attacks while pain is still mild. Acute medications usually work better when taken early in the attack, although patients need to be careful that they don't take the medication often enough to cause medication overuse headache.

\section{Choosing an acute medication for a specific patient}

Patients with migraine can be divided into a number of groups based on the severity of their migraine attacks, the medications they have tried before, and how well the medications that they have tried have worked for them. In this way, one can decide which medication would be best for an individual patient at a particular time.

Situation 1: For patients with severe attacks that often require bed rest it is best to try a triptan (with a medication to treat nausea if necessary). Subcutaneous sumatriptan $6 \mathrm{mg}$ is often best for severe attacks with early vomiting, or for severe attacks that do not respond to triptan tablets.

Situation 2: Patients with less severe attacks and who have not taken medications before for their migraines can try medications according to the following plan. For those who have tried some medications, they can be fitted into the plan after the first step or two, depending on what has been tried and how well it has worked. If a medication works quite well, there is no need to go on to the next steps. It is also important for patients to:

a. Learn about the various treatment options that are available, so they don't become discouraged when something they try does not work.

b. Understand that a medication for nausea (metoclopramide $10 \mathrm{mg}$ or domperidone $10 \mathrm{mg}$ ) can be added to all the acute migraine medications if needed for nausea.

Step 1: Individuals who have developed migraine for the first time can try one of ASA 1,000 mg, ibuprofen $400 \mathrm{mg}$, diclofenac potassium $50 \mathrm{mg}$, or naproxen sodium 500 - $550 \mathrm{mg}$. If they have a history of stomach ulcers or find that the NSAIDs cause side effects for them, they can try acetaminophen 1,000 mg. For patients with relatively severe attacks (but not usually requiring bed rest), a triptan can be prescribed by the doctor at the same time. The triptan can then be used as a rescue medication by the patient as necessary if the NSAID or acetaminophen occasionally fails. If the patient finds that the NSAID usually does not work well, the NSAID can be stopped and the triptan can become the patient's main medication.

Step 2: For patients who do not respond well to NSAIDs, a triptan is usually the best medication for use as their main acute migraine treatment. Some important points:

a. At least three different triptans should be tried (in different attacks) if the first triptan the patient tries does not work well. If it is working well, the patient should be pain free or almost pain free two hours after taking the medication, and be able to do usual activities with no significant side effects.

b. A triptan should be used to treat approximately three separate migraine attacks before deciding whether it is going to work well or not.

c. Intranasal triptans which are partially absorbed through the lining of the nose (for example zolmitriptan $5 \mathrm{mg}$ ) may work better than tablets for patients with nausea.

d. Orally dissolving tablets (wafers) may work better than regular tablets for patients with nausea that is worsened by using water to swallow regular tablets.

e. Triptans usually work best when taken early in the migraine attack while pain is still mild, although too frequent use (use on ten days a month or more) should be avoided. Patients with migraine with aura usually find it most helpful to take their triptan when the headache starts rather than during the aura itself, although triptan treatment during a typical migraine aura is safe. If patients find that taking a triptan during the aura is effective in treating their headache, they can continue to use their triptan in that way. 
f. When patients have relief of their migraine attack with a triptan, but the headache comes back within the next 24 hours, a second dose of the triptan usually works well.

g. If patients find that their headache often comes back within 24 hours after it has been relieved by a triptan, it may be helpful to switch to eletriptan, frovatriptan, or dihydroergotamine (DHE) from their current triptan. It may also be helpful to take 500-550 mg of naproxen sodium together with the triptan that they are using.

h. Patients with some severe migraine attacks and others that are less severe may find it helpful to use an NSAID for some attacks and a triptan for others.

Step 3: If triptans do not work very well for a patient, it may be helpful if the patient takes naproxen sodium $(500-550 \mathrm{mg}$ ) together with the triptan.

Step 4: For patients with severe attacks who usually respond well to a triptan-naproxen sodium combination, but have an occasional treatment failure, a rescue plan which may include a different rescue medication for use at those times may be helpful. Rescue medications that can be used include: prochlorperazine tablets or suppositories, occasional use of corticosteroids (such as prednisone), and occasional use of acetaminophen with tramadol or codeine. It is best for patients to discuss rescue medication options with their physician.

Step 5: For patients who do not respond satisfactorily to a triptan-naproxen sodium combination, the use of dihydroergotamine (nasal spray or self-injection), in future attacks combined with oral metoclopramide (if needed), may be helpful.

Step 6: Although not recommended for routine use in migraine, opioid analgesics (for example acetaminophen with codeine or tramadol) remain an option for patients who do not respond well to earlier treatment steps. These medications are also a treatment option for patients who do not respond to NSAIDs and who cannot take triptans because they have had a heart attack or stroke. Patients using medications with codeine or tramadol should:

a. Closely monitor how often they use them (using a headache diary).

b. Learn how to find and avoid their migraine attack triggers, and what lifestyle changes might make their migraines less frequent.

c. Consider whether a migraine preventive medication might be helpful for them.

In summary, by going through these steps patients can see where they fit in, and wh at medication they should try next.

\section{Menstrual migraine attacks}

Menstrual migraine attacks are migraine attacks that occur around the time of menstruation. For some women, these are their most severe attacks, and the ones that are the most difficult to treat. The treatment of menstrual migraine attacks may include:

a. The same treatments that are used for migraine attacks at any time, as discussed above. If the patient has many attacks at other times during the menstrual cycle as well, the usual preventive medications can be used. b. If patients have their migraine attacks mainly just before and during menstruation and don't respond well to NSAIDs or triptans used in the usual way, they can use naproxen sodium (500 - 550 mg) or frovatriptan $(2.5 \mathrm{mg})$ twice a day for six days, starting 2 days before menstruation is expected to start in order to prevent their menstrual migraine attacks.

c. If none of the options described above are effective, several types of hormonal treatments can be tried, but these are best discussed with the physician.

\section{Migraine during pregnancy}

Many patients with migraine improve during pregnancy, but medications are still often necessary. The best strategy is, however, to avoid use of medications during pregnancy if possible, especially during the first trimester, to avoid affecting the unborn baby. Non-medication treatments like avoiding migraine triggers (for example too much stress or too little sleep) and using relaxation exercises may be helpful. Patients who have questions about medication use in pregnancy can check the Motherisk website (www.motherisk.org/) or call Motherisk at the Hospital for Children (tel: 416-813-6780 or 1-877-439-2744). With regard to medications:

a. Acetaminophen is considered to be the safest painkiller for use during pregnancy. If acetaminophen is not helpful, occasional use of acetaminophen with codeine is also considered relatively safe.

b. NSAIDs (for example ibuprofen, naproxen sodium) should be used with caution during pregnancy. In the first three months of pregnancy, they may increase the risk of a miscarriage slightly. They should be avoided in the last three months because of effects on the baby. ASA should be avoided during pregnancy.

c. Triptans are relatively new drugs, and not recommended for routine use during pregnancy. Sumatriptan does seem relatively safe, with no effects on the baby, although it may increase bleeding during delivery. As experience is greatest with sumatriptan, if a triptan is being considered for use during pregnancy, sumatriptan is the one to use. It may be considered when other medications have failed, and migraine headaches are severe with major disability and/or vomiting.

d. Metoclopramide has not been associated with birth defects, and may be used during pregnancy. Domperidone should be avoided.

e. Ergotamine and dihydroergotamine should not be used during pregnancy.

\section{Migraine during breastfeeding}

Drugs are best avoided during breast feeding as well, but many medications are safe as outlined below.

a. Acetaminophen is considered safe during breastfeeding.

b. Ibuprofen is considered the safest NSAID during breast feeding. Diclofenac, ketorolac, and naproxen are also considered relatively safe, but ASA should be avoided.

c. Among the triptans, sumatriptan is considered safe during breast feeding. 
d. Among medications for nausea, metoclopramide, domperidone, dimenhydrinate, and prochlorperazine are all considered safe in breastfeeding.

e. Codeine in occasional doses is considered relatively safe, although it is best avoided if possible, especially if the infant is premature or less than one month old. It has on rare occasions caused serious problems for breast feeding infants.

\section{Conclusions}

There are many medications that can be helpful for acute migraine treatment. Treatment needs to be tailored for each patient. Sometimes several different medications need to be tried in order to find one that works best for a patient without causing too many side effects. It is best if patients learn about the treatment options and when to use them so that they can partner better with their physicians and pharmacists in the management of their headaches. The more patients learn about migraine treatment, the more successful they are likely to be. The Headache Network Canada website (www.headachenetwork.ca) is a very good source of information for patients with migraine. 